\title{
Análisis de implementación del Sistema Nacional de Telecomunicaciones en Emergencias como herramienta para gestión del riesgo: casos de Bogotá y Medellín
}

\author{
Analysis of the implementation of the National Telecommunications System \\ in Emergencies as a tool for risk management: cases from Bogotá and Medellín
}

\section{Análise de implementação do Sistema Nacional de Telecomunicações em Emergências como ferramenta para gestão do risco: casos de Bogotá e Medellín}

\author{
Gloria Amparo Rico Villegas ${ }^{*}$ | OmarVivas Cortés ${ }^{b}$ \\ ${ }^{\text {a }}$ https://orcid.org/0000-0002-6873-1227 Escuela de Ingenieros Militares - ESING, Bogotá, Colombia \\ ${ }^{\mathrm{b}}$ https://orcid.org/0000-0002-I 3 |3-6084 Universidad Externado de Colombia, Bogotá, Colombia
}

- Fecha de recepción: 2019-05-20

- Fecha concepto de evaluación: 2019-10-15

- Fecha de aprobación: 2019-11-16 http://dx.doi.org/ | 0.22335/rlct.vI I i3.917
Para citar este artículo / To reference this article / Para citar este artigo: Rico Villegas, G. A., \& Vivas Cortés, O. (2019). Análisis de implementación del Sistema Nacional de Telecomunicaciones en Emergencias como herramienta para gestión del riesgo: casos de Bogotá y Medellín. Revista Logos Ciencia \& Tecnología, I / (3), 68-8I. http://dx.doi.org// 0.22335/ rlct.vlli3.917

\begin{abstract}
RESUMEN
Este artículo presenta los problemas y las necesidades descubiertas en la investigación sobre la implementación del Sistema Nacional de Telecomunicaciones en Emergencias (SNTE), Decreto 2434 de 2015, en los casos de estudio de las ciudades de Bogotá y Medellín. El objetivo es determinar el grado de avance del SNTE entre las entidades encargadas de la gestión del riesgo de desastres. Las evidencias indican que hay un avance en cada ciudad objeto de estudio en cuanto al fortalecimiento de sus sistemas de comunicaciones en emergencias, pero no existe una articulación operativa que indique un avance sustancial en la implementación del SNTE en una escala supramunicipal. Como recomendaciones se plantean dos grandes desafíos políticos para que este proceso se lleve de una manera ordenada y sistemática, el primero es lograr que la información del SNTE sirva para definir esquemas de gobernabilidad de la gestión del riesgo para las ciudades; el segundo es la inclusión de los actores sociales y de sus redes para reducir el escenario de vulnerabilidad social vinculado con los desastres. Esta investigación es de carácter exploratorio con enfoque cualitativo, empleando el estudio de caso como técnica central probada.
\end{abstract}

Palabras clave: gestión del riesgo de desastres, prevención de desastres, respuesta ante emergencias, Sistema Nacional de Telecomunicaciones en Emergencias, categorías de comunicaciones en emergencias 


\section{SUMMARY}

This article presents the problems and needs discovered in the investigation on the implementation of the National Emergency Telecommunications System (SNTE), decree 2434 of 20I5, in the case studies related to the cities, Bogotá and Medellin. The objective is to determine the degree of progress of the SNTE among the entities in charge of disaster risk management. The evidence indicates that there is progress in each city studied in terms of strengthening their emergency communications systems, but there is no operational articulation that indicates a substantial advance in the implementation of the SNTE on an inter-municipal scale. As recommendations, two major political challenges are proposed so that this process is carried out in an orderly and systematic manner; the first is to ensure that the information from the SNTE helps to define governance schemes for risk management for cities; the second is the inclusion of social actors and their networks to reduce the social vulnerability scenarios linked to disasters. This research is exploratory in character with a qualitative approach, using the case study as a proven central technique.

Keywords: Disaster risk management, disaster prevention, emergency response, National Emergency Telecommunications System, emergency communications categories

\section{SUMÁRIO}

Este artigo apresenta os problemas e as necessidades descobertas na pesquisa sobre a implementação do Sistema Nacional de Telecomunicações em Emergências (SNTE), Decreto 2434 de 2015, nos casos de estudo das cidades de Bogotá e Medellín. O objetivo é determinar o grau de avanço do SNTE entre as entidades encarregadas da gestão do risco de desastres. As evidências indicam que há um avanço em cada cidade objeto de estudo em relação ao fortalecimento de seus sistemas de comunicações em emergências, mas não existe uma articulação operativa que indique um avanço substancial na implementação do SNTE em uma escala supra municipal. Como recomendações se buscam dois grandes desafios políticos para que este processo seja levado de maneira ordenada e sistemática; - primeiro é conseguir que a informação do SNTE sirva para definir esquemas de governabilidade da gestão do risco para as cidades; o segundo é a inclusão dos agentes sociais e de suas redes para reduzir o cenário de vulnerabilidade social vinculado com os desastres. Esta pesquisa é de caráter exploratório com enfoque qualitativo, empregando o estudo de caso como técnica central aprovada.

Palavras-chave: Gestão do risco de desastres, prevenção de desastres, resposta diante de emergências, Sistema Nacional de Telecomunicações em Emergências, categorias de comunicações em emergências

Los desastres generan afectaciones humanas, sociales y económicas que configuran un problema de desarrollo que es objeto de intervención pública desde la gestión del riesgo de desastres (en adelante GRD), utilizando para ello medios tales como el conocimiento, la capacitación y la difusión, el planeamiento territorial, la intervención física $y$, por supuesto, el desarrollo de tecnologías para las telecomunicaciones, que desempeñan un papel fundamental en la GRD y en el desarrollo de un país (Ortiz \& Valencia, 2017).

En este marco, las tecnologías de la información y las comunicaciones (TIC) son una herramienta que soporta los procesos de GRD (CRC, 20I6), al ser entendidas como aquellos recursos y programas que permiten acceder, producir, guardar, presentar y transferir información con la finalidad de facilitar los procesos de comunicación e intercambio de información para hacerlos cada vez más rápidos y eficientes (Ministerio de las Tecnologías de la Información y las Comunicaciones [MinTIC], 20I5). La comunicación es vital para las actividades de sensibilización y prevención, comunicar el estado de las amenazas, generar alertas, así como para el manejo adecuado de los desastres (Unión Internacional de Telecomunicaciones [UIT], 2003). 
A partir de esta importancia de las TIC para salvar vidas en situaciones de emergencia, varios países, entre los que se incluye Colombia ratificaron, el Convenio de Tampere, que entró en vigor a partir del año 2005. Los países firmantes han desarrollado diferentes medidas para sus propias redes nacionales de comunicaciones en emergencias. España, por ejemplo, basa su modelo en el Sistema de Radiocomunicaciones Digitales de Emergencia del Estado (SIRDEE), "un sistema que tiene relación con los sistemas de comunicación de seguridad del Estado y con las diferentes redes públicas y privadas que existen en el país" (Fontic-Iteco, 2013, p. 40).

Perú, otro de los países firmantes, cuenta con dos redes nacionales para facilitar la comunicación entre autoridades del Estado: En primera instancia está la Red Especial Terrestre de Comunicaciones en Emergencias (RECSE) que se soporta "en las redes de servicios públicos de telefonía fija y móvil convencionales" (Fontic-Iteco, 2013, p. 191). En segunda instancia y solo cuando no están disponibles los servicios de telefonía fija y móvil, se activa la Red Especial Satelital de Comunicaciones en Emergencias (Redsat) (Fontic-Iteco, 2013). Adicionalmente, cuenta con reglamentación para que toda la infraestructura de las empresas operadoras de telecomunicaciones esté a disposición de las autoridades ante una emergencia.

Asimismo, en Argentina, otro de los países sudamericanos firmantes del Convenio de Tampere, se cuenta con una entidad que coordina la atención en emergencias y desastres (Sifem), la cual maneja una red principal de comunicaciones, que está conformada por las redes de telefonía fija y móvil, las cuales son interoperables y permiten la interconexión entre los usuarios de distintas redes. "Como red complementaria y alterna se tienen los equipos de los radioaficionados, con sistema de HF con cubrimiento nacional y VHF/UHF para cubrimiento regional" (FonticIteco, 20I3, p. 209).

En Colombia, el Ministerio de Tecnologías de la Información y las Comunicaciones (MinTIC) es la entidad encargada de promover el acceso, el uso efectivo y la apropiación masiva de las TIC a través de la creación de políticas y programas que promueven el acceso eficiente a estas, en igualdad de oportunidades para todos los habitantes del territorio nacional (Congreso de la República de Colombia, 2009). Atendiendo estos preceptos, el Gobierno nacional expidió el Decreto 2434 de 2015 que creó el Sistema Nacional de Telecomunicaciones de Emergencias (SNTE) como parte del Sistema Nacional de Gestión del Riesgo de Desastres (Decreto 2434, 2015). El SNTE busca facilitar, apoyar y fortalecer las comunicaciones requeridas en los procesos de GRD, indicar las directrices para la prestación de los servicios de telecomunicaciones en situaciones de emergencias y además establecer los lineamientos de los sistemas de información dentro de las políticas generales de Gobierno en Línea definidas en el Decreto 2573 de 2014 (Decreto 2434, 2015).

A partir de estudios como el "Análisis de alternativas para la formulación, diseño y modernización de la Red Nacional de Telecomunicaciones de Emergencia, y el Estudio de Vulnerabilidad y Riesgo de las redes e infraestructura de Telecomunicaciones en zonas vulnerables expuestas a eventos naturales desastrosos", el MinTIC orientó el desarrollo del SNTE en busca de dar una respuesta articulada a las necesidades del país en la materia (Fontic; Consorcio Itelca-STI, 20/3). Para ello se analizaron los tres referentes internacionales mencionados junto con otros ocho casos, es decir, once países en total, retomando once aspectos para el análisis ${ }^{1}$ que permiten dar cuenta de buenas prácticas para la implementación de redes de telecomunicaciones a escala nacional.

Al ser un sistema reciente en el país, aún no se cuenta con estudios que hayan evaluado el estado de implementación del SNTE en el nivel nacional, ni local; por tal razón la investigación analizó la implementación local en Bogotá y Medellín, ciudades representativas y que tienen avances en la materia (Departamento Nacional de Planeación [DNP], 2014). Con esta investigación se profundiza en el conocimiento de las necesidades de las entidades territoriales frente a las telecomunicaciones en la GRD; ello atiende a la necesidad de alcanzar el desarrollo institucional a partir del conocimiento de las situaciones institucionales, máxime cuando hay un gran número de habitantes expuestos a las amenazas del territorio (Departamento Administrativo Nacional de Estadística [DANE], 2018).

La hipótesis que orienta la investigación es: tanto en Bogotá como en Medellín no ha sido posible la implementación

I (I) Arquitectura de la red de emergencias; (2) cobertura de la red de emergencias; (3) tecnologías empleadas en la red de emergencias; (4) bandas de frecuencias utilizadas en la red de emergencias; (5) servicios soportados por la red de emergencias; (6) trayectos cableados; (7) clases de redundancia implementadas en la red de emergencias; (8) conectividad de la red de emergencias; (9) clases de usuarios de la red de emergencias; (10) entidades conectadas; (II) administración y operación de la red de emergencias. 
articulada del SNTE, lo que dificulta la aplicación del desarrollo normativo. Así, la pregunta de investigación es: ¿qué factores dificultan la implementación del Sistema Nacional de Telecomunicaciones en Emergencias (SNTE) para la gestión del riesgo de desastres, para los casos de Bogotá y Medellín? De esta manera, se propuso como objetivo general evidenciar los problemas de articulación de la implementación del SNTE de acuerdo con las necesidades expresadas y los avances alcanzados por las entidades competentes para la GRD en estas dos ciudades.

Para ello se realizó una investigación exploratoria con enfoque cualitativo, empleando el estudio de caso como técnica central probada (Ávila, Vivas, Herrera, \& Jiménez, 2016). Las herramientas para la recolección de información fueron la entrevista semiestructurada, la observación directa y el análisis documental y normativo. Las entrevistas semiestructuradas se aplicaron a cinco actores claves de las entidades competentes para la GRD en cada ciudad, es decir, el Instituto Distrital de Gestión de Riesgos y Cambio Climático (Idiger), en el caso de Bogotá, y el Departamento Administrativo de Gestión del Riesgo de Desastres (DAGRD) en Medellín, así como algunos representantes de entidades del nivel nacional que hacen parte del SNTE, todos ellos conocedores de la temática y funcionarios con responsabilidad directa en la implementación del SNTE en sus respectivas entidades territoriales ${ }^{2}$. Al trabajar desde un enfoque cualitativo, las entrevistas se manejaron con el programa AtlasTi para generar categorías de análisis de acuerdo con los objetivos propuestos en la investigación.

\section{Gestión del riesgo y comunicaciones} en emergencias: contexto nacional

Según informaba el Banco Mundial,

el $86 \%$ de la población colombiana se localiza en zonas de amenaza sísmica alta y media, el $12 \%$ del territorio nacional se encuentra localizado en áreas con una mayor susceptibilidad a inundarse, el $18 \%$ del territorio nacional se encuentra localizado en zonas de amenaza muy alta y alta por movi-

2 Es importante resaltar que, al ser una investigación cualitativa, no resulta tan relevante la cantidad de actores entrevistados, sino su rol en la problemática a investigar y la calidad de la información brindada, así como su veracidad. Debido a que el objetivo central de la investigación es identificar el avance en la implementación del SNTE y la articulación institucional, los cinco actores entrevistados son las personas competentes directamente para la implementación y desarrollo de esta y otras políticas en materia de GRD. mientos en masa, principalmente en los departamentos de la región Andina. (UNGRD, 20I 5a, p. 2).

En estas zonas se concentra la mayor cantidad de población del país.

Para el año 2012 fue expedida la Política Nacional de Gestión del Riesgo de Desastres,

indispensable para asegurar la sostenibilidad, la seguridad territorial, los derechos e intereses colectivos, mejorar la calidad de vida de las poblaciones y las comunidades en riesgo $y$, por lo tanto, está intrínsecamente asociada con la planificación del desarrollo seguro, con la gestión ambiental territorial sostenible, en todos los niveles de gobierno y la efectiva participación de la población (Congreso de la República de Colombia, 20I2, p. I).

Así las cosas, desde una perspectiva político-normativa, la GRD en Colombia es concebida como:

Un proceso social orientado a la formulación, ejecución, seguimiento y evaluación de políticas, estrategias, planes, programas, regulaciones, instrumentos, medidas y acciones permanentes para el conocimiento y la reducción del riesgo y para el manejo de desastres, con el propósito explícito de contribuir a la seguridad, el bienestar, la calidad de vida de las personas y al desarrollo sostenible (Congreso de la República de Colombia, 2012).

Al ser un proceso ligado a la búsqueda del desarrollo sostenible de la población y el territorio, la GRD debe ser planificada, concertada, participativa e integral (Díaz, Chuquisengo, \& Farradas, 2005), permitiendo así, en palabras de Hardaker et al.y Babcock (citados por Medina, 20I4) "la aplicación sistemática de políticas de gestión, procedimientos y prácticas con el fin de identificar, analizar, evaluar, tratar y realizar el seguimiento del riesgo" (Medina, 20I4, p. 59).

Desde la Ley 1523 se adaptó el marco conceptual que comprende la GRD como la interrelación de tres procesos. El primer proceso, de conocimiento, se refiere a

la identificación de escenarios de riesgo, el análisis y evaluación del riesgo, el monitoreo y seguimiento del riesgo y sus componentes y la comunicación para promover una mayor conciencia del mismo que alimenta los procesos de reducción del riesgo y de manejo de desastre (Congreso de la República de Colombia, 2012, p. 4). 
En este proceso, las telecomunicaciones permiten la divulgación de información y la sensibilización a la población frente al riesgo (CRC, 2016, p. 4).

El segundo proceso, de reducción del riesgo, "está compuesto por la intervención dirigida a modificar o disminuir las condiciones de riesgo existentes, entiéndase: mitigación del riesgo y a evitar nuevo riesgo en el territorio, entiéndase: prevención del riesgo" (Congreso de la República de Colombia, 20I2, p. 4). El tercer proceso, de manejo de desastres está "compuesto por la preparación para la respuesta a emergencias, la preparación para la recuperación pos desastre, la ejecución de dicha respuesta y la ejecución de la respectiva recuperación, entiéndase: rehabilitación y recuperación" (Congreso de la República de Colombia, 20I2, p. 4).

Los equipos de telecomunicaciones móviles, los computadores, la radio, la televisión, el internet, las redes de comunicaciones, entre otros, constituyen elementos que apoyan y fortalecen los procesos GRD, al mejorar el flujo de información vital para prestar una adecuada asistencia antes, durante y después de una catástrofe (MinTIC, 20I4).

Ante las distintas formas de emplear las telecomunicaciones en la GRD, la Comisión de Regulación de Comunicaciones (CRC), basándose en los desarrollos conceptuales de la UIT, definió cuatro categorías de comunicaciones que corresponden a las diferentes "instancias de comunicación entre los actores involucrados en las fases de prevención, atención, y recuperación" (CRC, 2016, p. 17). El Decreto 2434 de 2015 define las tipologías de comunicación de la siguiente forma:

I) Autoridad-autoridad: comunicación entre las autoridades encargadas de atender las situaciones de emergencia, con ella facilitan las operaciones de recuperación, la restauración de la infraestructura comunitaria esencial y la adaptación de las primeras medidas que permitan la recuperación a largo plazo (Decreto 2434, 2015).

2) Autoridad-individuo: comunicación que dirige las autoridades hacia los individuos a efectos de prevenir o mitigar las condiciones de riesgo y orientar la respuesta en las situaciones de emergencia, entran en esta categoría los sistemas de alerta temprana. El contenido puede ser, entre otros, información, orientación 0 instrucciones dirigidas a una comunidad que se puede ver afectada o se afectó por una emergencia, calamidad pública o desastre (Decreto 2434, 2015).

3) Individuo-autoridad: telecomunicaciones de los individuos hacia las autoridades con el fin de informar acerca de una situación de emergencia o buscar ayuda para su mitigación. Hacen parte de esta categoría las llamadas que genera un individuo a un centro de atención de emergencias (CAE) mediante el número único nacional de emergencias 123 (Decreto 25 de 2002) o utilizando otros medios como serían el video, el correo electrónico, los mensajes de texto y la mensajería instantánea, entre otros (Decreto 2434, 2015).

4) Individuo-individuo: telecomunicaciones que se realizan entre la población en situaciones de emergencia o desastre, generan alta demanda de recursos de telecomunicaciones y pueden llegar a congestionar las redes públicas (Decreto 2434, 2015).

En el contexto de la Ley 1523 de 2012 y como parte del Sistema Nacional de Gestión del Riesgo de Desastres (en adelante SNGRD), se estructuró la política del SNTE con el fin de establecer las directrices para la prestación de los servicios de telecomunicaciones en situaciones de emergencias, conforme es indicado en la siguiente ilustración.

EI SNTE es coordinado por la Unidad Nacional para la Gestión del Riesgo de Desastres (UNGRD), entidad de la rama ejecutiva, y en los niveles departamental y
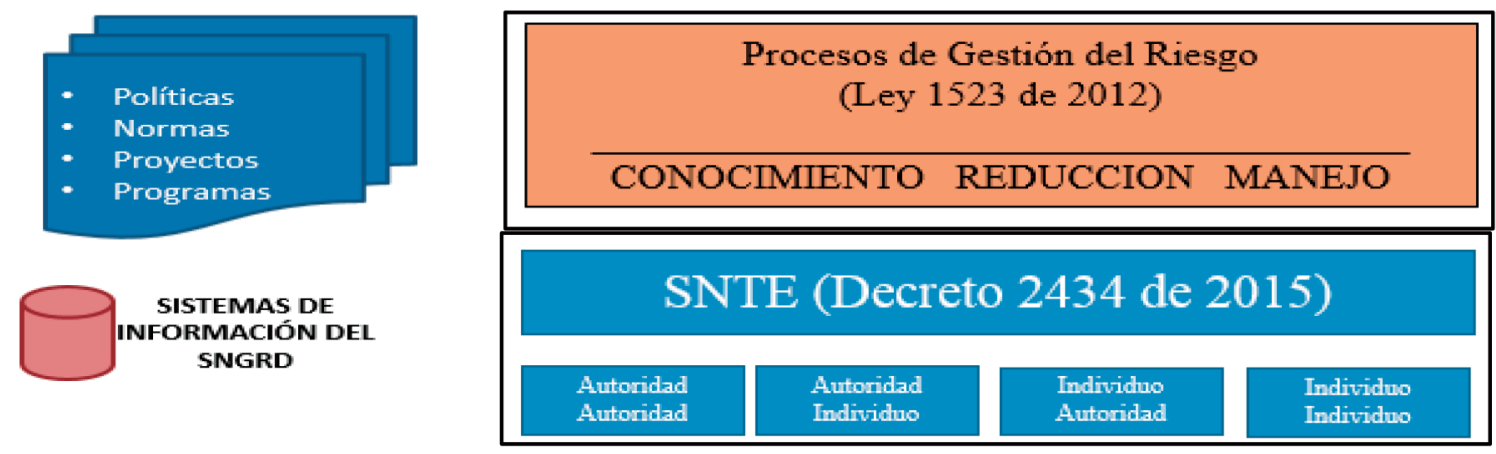

Figura I. Estructura del SNTE en el marco del SNGRD

Fuente: elaboración propia con base en la Ley 1523 de 2012 (Decreto 2434, 20I5) (CRC, 2016). 


\section{SNTE: componentes actuales}
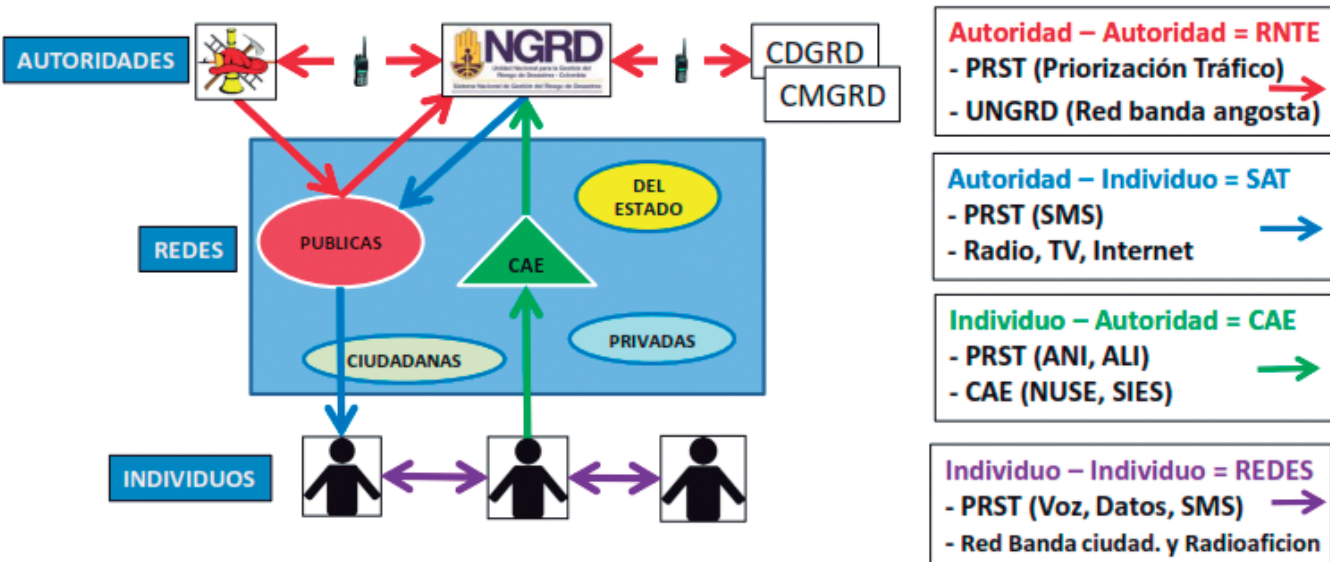

Figura 2. Componentes del SNTE

Fuente: Regulación para promover el desarrollo e implementación del Sistema Nacional de Telecomunicaciones de Emergencias en Colombia (CRC, 2016, p. 39).

municipal, por los Consejos Departamentales y Municipales de Gestión del Riesgo de Desastres (CDGRG, CMGRD), presididos por las autoridades de cada entidad territorial.

La política del SNTE prescribe acciones específicas para fortalecer las telecomunicaciones mediante objetivos centrales que se materializan en proyectos dentro de los cuales se puede observar que aún no se detallan las acciones para dar viabilidad a la red propuesta en los niveles departamentales y municipales, como se muestra en la tabla $\mathrm{I}$.

Los proyectos para la implementación del SNTE dependen de la autoridad nacional, por ser iniciativas normativas generales (Resolución 4972, 2016). El primer objetivo y los proyectos relacionados, hacen referencia directa a competencias de las entidades municipales, como son los Sistemas de Alerta Temprana (SAT) y la atención en los Centros de Atención de Emergencias, donde se reciben y despachan las llamadas de emergencia al número 123 , proyectos que se han visto reflejados en los instrumentos de planificación de las dos ciudades objeto de estudio, tanto en planes de desarrollo, como planes de gestión del riesgo, garantizando su operatividad (Alcaldía Municipal de Medellín, 2016;Alcaldía Mayor de Bogotá, 2016).

\section{Situación observada: nivel nacional}

Las entidades llegan a articular sus actividades a partir de las normas y las instancias de coordinación allí definidas y a través de planes, proyectos e inversiones, aspectos a valorar para el primer nivel de implementación de la política, el nivel nacional. El Decreto 2434 de 2015 definió un plazo de seis meses para que MinTIC realizara la reglamentación en su sector de competencia (MinTIC, 20I8, p. I); sin embargo, durante la vigencia 2016 no se expidió la resolución por la cual se regularía el SNTE postergando así la implementación real (MinTIC, 20I8).

Por otra parte, el Decreto 2434 estipuló que, para el año 2017 , los proveedores de telecomunicaciones debían contar con los mecanismos para la identificación y localización de abonado llamante, así como con mecanismos de priorización de comunicaciones autoridad-autoridad para llamadas dentro de la propia red, y en el año 2018 para llamadas que involucraran otras redes. Empero, según informe del MinTIC a la Contraloría General de la República, el avance a 2018 era apenas del $10 \%$, debido a dificultades en la gestión para formular instrumentos de planeación y política pública con "metas que muestren resultados reales y permitan la operación del sistema en caso de emergencias y desastres, así como para obtener la participación y el compromiso de las diferentes entidades y actores que lo integran" (MinTIC, 20I8, p. 2).

El avance más representativo en materia regulatoria ha sido la Regulación para promover el desarrollo y la implementación del SNTE en Colombia, publicada por la CRC en mayo del año 2016 (CRC, 20I6). La ANE, por su parte, desde 2017 está tramitando un proyecto para actualizar la normatividad vigente en cuanto a las frecuencias en banda angosta destinadas a atención de emergencias y desastres 
Tabla I. Objetivos y proyectos en la Política del SNTE

\section{Objetivo}

Proyectos

Facilitar, apoyar y fortalecer las comunicaciones requeridas en los • Implementar la Red Nacional de Telecomunicaciones de Emergencias procesos de la gestión del riesgo de desastres

- Implementar envío de mensajes de alerta temprana sobre las redes de telefonía móvil

- Implementar servicio de localización del abonado llamante que se comunica con los Centros de Atención de Emergencias (CAE)

Coordinar la intervención del sector de telecomunicaciones en los procesos de conocimiento del riesgo, reducción del riesgo y manejo de desastres

Establecer directrices para la prestación de los servicios de telecomunicaciones en situaciones de emergencias
Expedición de la normatividad mediante la cual se regula el SNTE

- Seguimiento al cumplimiento de la normatividad mediante la cual se regula el SNTE

- Expedición de la normatividad específica para coordinar la intervención del sector de telecomunicaciones en situaciones de emergencias

- Seguimiento al cumplimiento de la normatividad específica para coordinar la intervención del sector de telecomunicaciones en situaciones de emergencias

Coordinar con la Agencia Nacional del Espectro (ANE) la planeación del espectro radioeléctrico necesario para la gestión del riesgo, conforme a las recomendaciones de los organismos nacionales e internacionales

Realizar la atribución, adjudicación y asignación de frecuencias cuando se requiera

Orientar, entre otros, los aspectos normativos de las telecomunicaciones - Expedición de la normatividad mediante la cual se regula el SNTE que contribuyan al funcionamiento del Sistema, con el apoyo de las enti- - Seguimiento al cumplimiento de la normatividad mediante la cual se dades competentes regula el SNTE

Fuente: Política Pública del Sistema Nacional de Telecomunicaciones en Emergencias.Versión I.0., 2016, p. 83)

en Colombia (ANE, 2017), acciones alineadas con la RNTE en cuanto al uso de las redes VHF que se tienen en reserva para la respuesta ante emergencias (ANE, 20I8).

Acerca de la planeación, la UNGRD viabilizó programas y proyectos dentro del Plan Nacional de Gestión del Riesgo de Desastres 2015-2050, orientados hacia el fortalecimiento de la conectividad "entre las entidades territoriales, las entidades nacionales y la UNGRD a través de diversas redes y medios (HF, VHF, tecnología LTE)" (UNGRD, 20I5b, p. 5I). Sin embargo, en el trabajo de campo no fue posible identificar acciones concretas para viabilizar la RNTE dentro del SNTE, u otras acciones específicas con responsables y presupuestos, que fortalezcan el uso de TIC en la GRD en escalas municipales o locales.

El Fondo Nacional de Gestión del Riesgo de Desastres es la principal fuente de financiación para todas las actividades en los tres procesos de la GRD, y toda la ordenación del gasto está a cargo del director de la UNGRD (UNGRD, 2019). Para asignar recursos del fondo hacia la atención de eventos que presenten declaratoria de calamidad pública, la UNGRD consolida la información que reportan los consejos municipales y departamentales de GRD (UNGRD, 20I5c).

No obstante, para desplegar la infraestructura de comunicaciones requerida a nivel nacional para la GRD, es necesaria la financiación por parte de instituciones centralizadas, pues mientras la gran mayoría de entidades territoriales no disponen de recursos (DNP, 20I8), las ciudades como Bogotá y Medellín, entre otras, cuentan con sistemas que pueden conectarse a la RNTE a través de infraestructuras de interface y OPEX que el MinTic puede financiar (Acosta, 2019).

De esta forma, un avance para la consolidación de la RNTE y el SNTE sería su inclusión en programas o proyectos específicos del Plan Nacional del Desarrollo (PND 2018-2022), considerando que este es el instrumento por excelencia para organizar el presupuesto público sectorial a nivel nacional, a partir del cual los departamentos y municipios orientan sus propios procesos de planificación, con recursos incluidos, hacia la implementación de esta y otras políticas nacionales.

En las bases del PND se encontró que se plantearon proyectos para dotar de equipos y redes de comunicación al país, mas no se observa una programación directa de actividades y recursos para enmarcar esta dotación en el desarrollo de la RNTE. Si bien no es la única vía para programar actividades concretas que contribuyan a la implementación del SNTE, sí garantizaría un mayor nivel de articulación entre los distintos niveles de gobierno, así como los distintos sectores para contribuir a un desarrollo integrado del SNTE, acorde con las competencias, los recursos y las capacidades de cada entidad territorial. 


\section{Situación observada: caso Bogotá}

La institucionalidad para la GRD en la ciudad de Bogotá incorporó la mitigación y adaptación al cambio climático, dando origen al Instituto Distrital de Gestión de Riesgos y Cambio Climático (en adelante Idiger) como entidad rectora de la política pública para la GRD en el distrito. Asimismo, se creó el Sistema Distrital de Gestión de Riesgo y Cambio Climático (SDGR-CC) que articula a las entidades involucradas (Idiger, 20I6).

El Consejo Distrital de Gestión de Riesgos y Cambio Climático lo conforman los secretarios de despacho de los sectores administrativos de coordinación, el director (a) del Idiger, quien asume la secretaría técnica, y el alcalde mayor o su delegado, quien lo preside. Cuando se convoca para abordar temas de preparación, respuesta o rehabilitación ante situaciones de emergencia, hacen parte del consejo los directores o gerentes de las siguientes entidades: Empresa de Acueducto y Alcantarillado de Bogotá, Empresa de Energía de Bogotá, Empresa de Telecomunicaciones de Bogotá, Unidad Administrativa Especial Cuerpo Oficial de Bomberos, Policía Metropolitana, Corporación Autónoma Regional de Cundinamarca, Cruz Roja Colombiana Seccional Bogotá y la Defensa Civil Colombiana Seccional Bogotá.

\section{Comunicaciones autoridad-autoridad}

Como la GRD es competencia de cada entidad territorial según sus recursos y capacidades, según la Ley 1523, Bogotá creó un sistema cerrado en el nivel local, con poca participación directa del orden nacional excepto en situaciones que ameriten una declaratoria de calamidad pública, en cuyo caso se activa el Consejo Distrital. Por esta razón, la información de la GRD consolidada en el Sistema de Información para la Gestión del Riesgo y Cambio Climático de Bogotá (SIRE) no es reportada directamente a la UNGRD. Además, los módulos para la recepción de información a nivel nacional son diferentes a los datos producidos desde el SIRE. Sin embargo, el portal en línea de la Alcaldía actualiza la información relacionada con la GRD, tanto para entidades como para la ciudadanía en general.

Para facilitar la coordinación entre todas las instituciones e instancias que intervienen en el SDGR-CC se ha establecido una Red Distrital de Comunicaciones para Emergencias, con equipos de radio para comunicación en bandas VHF y UHF.

La funcionalidad de la red se soporta en diez canales de radios y una central en la oficina principal del Idiger.Adicionalmente cuenta con cinco repetidoras portátiles, que brindan funcionalidad de suplencia a los sistemas en caso de alguna falla (Idiger, 2015, p. 152).

En caso de requerirse el concurso de las distintas agencias competentes, el director del Idiger activa el Centro de Operaciones de Emergencia, donde se reúnen las autoridades y desde allí se activa el Puesto de Mando Unificado (PMU) que se establece en el lugar de los hechos (Idiger, 2017b). En el PMU se realiza la comunicación directa entre entidades, siguiendo lineamientos previamente brindados por las cadenas de mando de niveles superiores, los cuales permanecen en comunicación a través de la telefonía móvil y/o los radios de la red distrital (Idiger, 2017a).

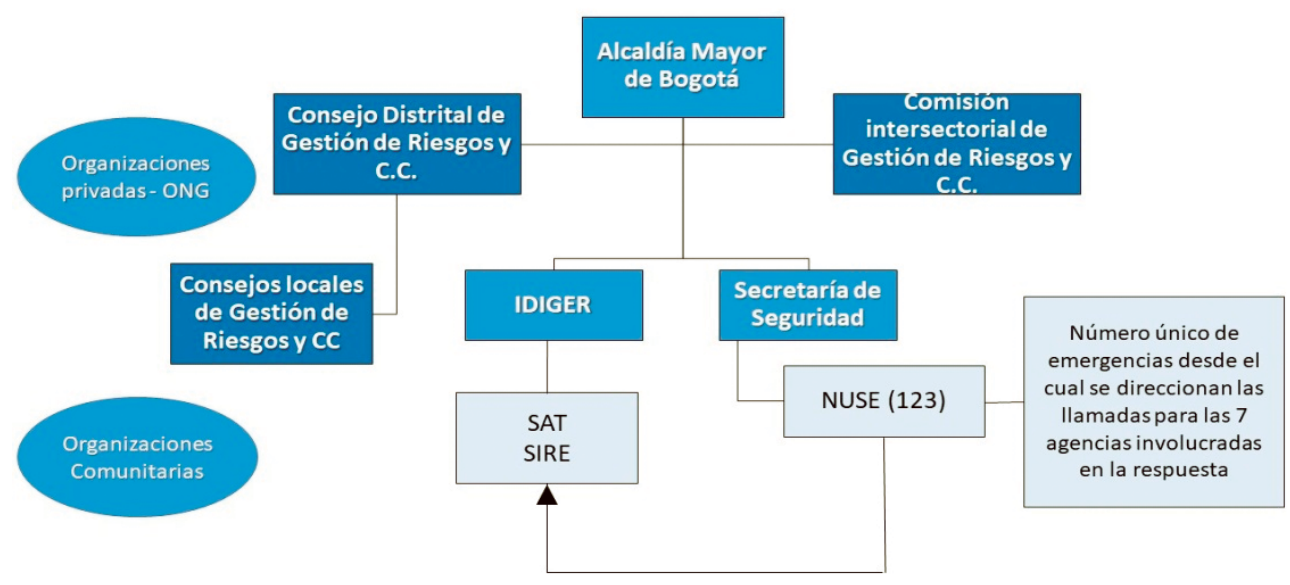

Figura 3. Sistema para la gestión del riesgo de desastres en Bogotá

Fuente: elaboración propia con base en el Decreto 579 de 2015 y Acuerdo 007 de 2016, Bogotá. 
El respaldo para una respuesta coordinada se encuentra en la Estrategia Distrital de Respuesta, la cual contiene los protocolos distritales de soporte, que activan funciones de información pública y de enlace interinstitucional y multiescalar para el manejo de emergencias y desastres (Idiger, $2017 b$ ), de manera que cada entidad cuenta con lineamientos precisos para responder ante la llamada o el evento de emergencia, y comunicarse con las entidades competentes.

A nivel directivo, la comunicación para la atención de emergencias no siempre se realiza a través de sistemas formales, por lo que frecuentemente se hace uso de la telefonía móvil, especialmente chat de WhatsApp, y en un caso de requerir toma de decisiones, como se mencionó, se convoca al Consejo Distrital (Idiger, 2017 a).

\section{Comunicaciones individuo-autoridad}

El Numero Único de Seguridad y Emergencias (NUSE) 123 es el sistema distrital donde se reciben y direccionan las llamadas de emergencia que realiza la ciudadanía. Está bajo la dirección y coordinación de la Secretaría de Seguridad y Convivencia. Esto ha permitido que la recepción de la primera llamada sea realizada por personal civil capacitado para el adecuado direccionamiento, $y$ las agencias competentes se articulan por medio de sus respectivas salas de comunicaciones, a través de un enlace permanente entre los respectivos sistemas de información y el Procad (Sistema de Información del NUSE) (Idiger, 20I5).

Sin embargo, aún se tiene una gran dificultad y es el hecho de que los sistemas utilizados por las agencias de despacho no son completamente compatibles con el sistema de articulación central (Procad-NUSE),

lo cual se materializa en vacíos de la información disponible para algunas agencias con respecto a determinados eventos. Las deficiencias en el flujo de la información afectan directamente la notificación, el despacho y seguimiento de los recursos disponibles para atender las emergencias, calamidades y/o desastres. (Idiger, 2015, p. 213)

Esta dificultad está siendo sorteada con la modernización del Centro de Atención de Emergencias, denominado C4, el cual se inauguró en el año 2018 y cuenta con tecnología de punta para una adecuada recepción de llamadas y un direccionamiento oportuno, con retroalimentación de estas. Se espera que esta inversión permita contrarres- tar la saturación del NUSE debido a la gran cantidad de llamadas falsas realizadas ${ }^{3}$.

Está en proceso la implementación del sistema para la plena localización del abonado llamante, pues a pesar de ser una acción prioritaria dentro de la política del SNTE, no había sido prioridad para el distrito (Vargas, 2019). Sin embargo, se ha observado que este servicio permite generar acciones para sancionar el mal uso de la línea 123, y en la actual administración se ha consolidado un trabajo más cercano con el MinTIC para lograr esta meta (ElTiempo, 2018; Rodríguez, 2019).

\section{Comunicaciones autoridad-individuo}

El Sistema Distrital de Alertas Tempranas (SAT) hace parte del Sistema de Información para la Gestión del Riesgo y Cambio Climático (SIRE). El portal web del SIRE brinda información relacionada con cambio climático, riesgo tecnológico, amenaza por sismo, aglomeración, inundación, incendio forestal y deslizamiento; integra además diferentes sistemas creados en el marco de la Ley 1523 de 2012, los cuales son:

- Sistema Único de Aglomeración de Público

- Sistema Único de Registro de Reasentamientos

- Sistema Único de Registro Escolar

- Sistema de Transporte Vertical

- Emergencias

- Guías de Preparación para Respuesta a Emergencias

- Experiencias Educativas

Cada uno de estos sistemas publica información en el portal web del SIRE, que brinda acceso al público en general. EI SAT, a su vez, se alimenta de cuatro redes de información: la Red Hidrometereológica de Bogotá, el Monitoreo de Tormentas Eléctricas, la Red de Acelerógrafos de Bogotá y el SAT Comunitario (Idiger, 2015). Este último hace parte de una perspectiva de la gestión del riesgo con un fuerte énfasis en la capacitación y el empoderamiento de actores comunitarios, quienes en algunos casos conocen mejor las redes de actores del vecindario que los técnicos y expertos externos, de manera que pueden potenciar activamente las relaciones comunales en función de la gestión del riesgo (Idiger, 20I5).

Los mensajes de alerta que se emiten a la comunidad, a partir de la información consolidada del SAT, utilizan todos los medios de comunicación disponibles: videos, pronósticos

\footnotetext{
3 El Nuevo código de policía (Ley I80I de 2016) establece sanciones para las personas que realicen llamadas falsas a la línea de emergencias, dentro de las que se incluye el pago de multas por medio de la factura telefónica (El Tiempo, 2018).
} 
del tiempo e informes de lluvias que se transmiten en las redes sociales, además de los boletines a los medios de comunicación. Se está trabajando en la mensajería de texto territorializada, para comunicar a la población cuando haya interferencias en los medios usuales: redes sociales, WhatsApp. Estas comunicaciones se encuentran contenidas en la mencionada Estrategia Distrital de Respuesta, a través de los protocolos distritales para los Sistemas de Alerta Temprana (PDSAT) (Idiger, 20I5).

En síntesis, Bogotá cuenta con un sistema institucional que integra las telecomunicaciones para la GRD. En aras de articularse a un SNTE, se requiere una plataforma que sea interoperable con las redes internas, facilitando el equilibrio costo-beneficio frente a las inversiones recientes. En cuanto a las categorías de telecomunicaciones en emergencias, la comunicación individuo-individuo es aquella que requiere mayor intervención de las autoridades regulatorias, pues no se logró hallar información de acciones o iniciativas al respecto. Las categorías restantes se han logrado manejar desde las competencias distrita- les, presentando desarrollos importantes con sus debilidades, fortalezas y oportunidades de mejora.

\section{Situación observada: caso Medellín}

El antecedente del Sistema Actual de Gestión del Riesgo (SMGRD) se referencia en el Acuerdo 059 de 201 I, donde se crea el Departamento Administrativo de Gestión del Riesgo de Emergencias y Desastres (DAGRD). A raíz del cambio de la normatividad con la Ley I523, en la ciudad de Medellín se consolidó un Sistema Municipal de Gestión del Riesgo de Desastres, que cuenta con instancias directivas e instancias de coordinación, en las que el DAGRD, al igual que en Bogotá, cumple tanto funciones centrales, como funciones complementarias desde su competencia técnica.

El CMGRD es la "instancia superior de coordinación, orientación, asesoría, planeación, seguimiento y evaluación, destinado a garantizar la efectiva articulación de los

\section{Alcaldía Municipal}

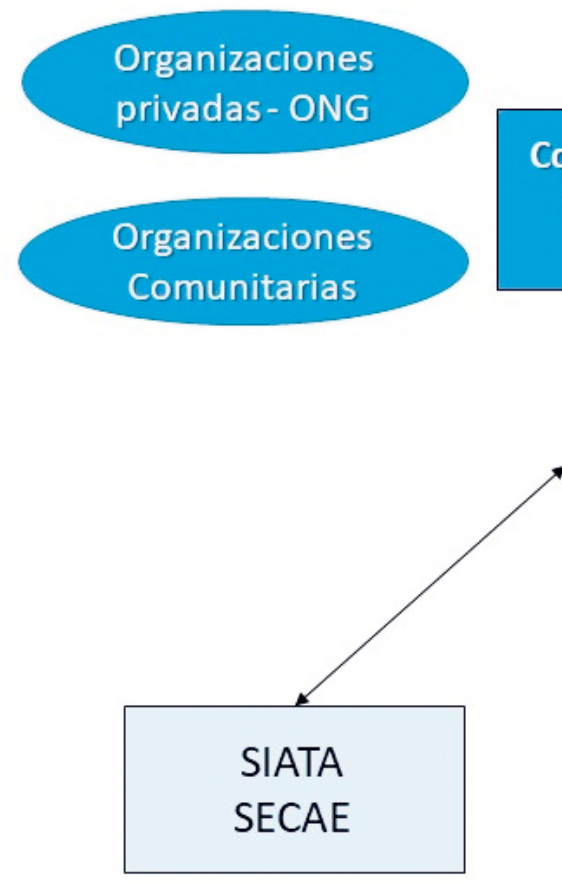

Consejo Municipal para la Gestión del Riesgo de Desastres (CMGRD)
Comisiones sectoriales

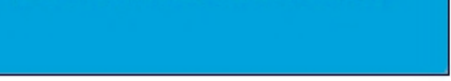

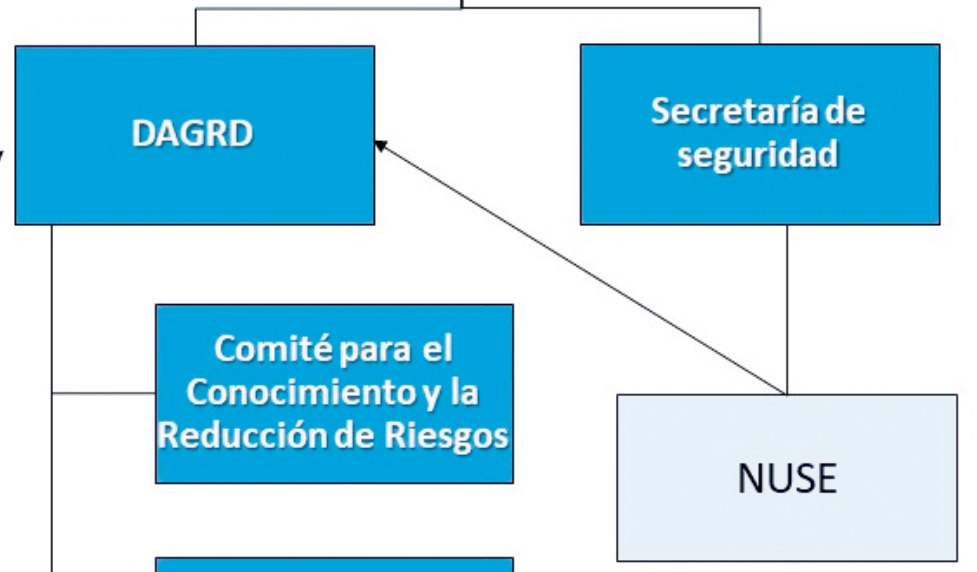

Comité Municipal

para el Manejo de

Desastres 
procesos de conocimiento, reducción del riesgo y el manejo de desastres..." (Decreto 1240, 2015) Por su parte, el DAGRD a través del Comité Municipal para el Conocimiento y la Reducción del Riesgo de Desastres es el encargado de orientar la formulación, ordenamiento territorial, planificación del desarrollo y adaptación al cambio climático relacionados con el conocimiento y la reducción de riesgos. Otra función asignada consiste en ser asesores de la rehabilitación y la reconstrucción, las intervenciones correctivas y la orientación de la formulación del Plan Municipal para la Gestión del Riesgo de Desastres.

El Comité Municipal para el Manejo de Desastres tiene como función orientar la formulación de políticas que fortalezcan el manejo de desastres, la calamidad pública y las emergencias; así mismo asesora la respuesta a situaciones de desastres, coordina con el Comité de Conocimiento y Reducción del Riesgo la reconstrucción en situación de desastre para que no se reproduzcan las condiciones de vulnerabilidad, y orienta la formulación, implementación, seguimiento y evaluación de la Estrategia Municipal para la Respuesta a Emergencias y los Planes de Acción Específicos de Recuperación (Decreto 1240, 2015).

De forma similar a Bogotá, el CMGRD es presidido por el alcalde municipal de Medellín, o su delegado; sin embargo, en Medellín el director del DAGRD asume el rol de presidente en ausencia del alcalde. Además, dicho comité está conformado por distintas secretarías de la administración municipal, y dependiendo del caso, el gerente de Empresas Públicas de Medellín, el Gerente de la Empresa de Transporte Masivo del Valle de Aburrá (Metromed) o su delegado, el director de la Corporación Autónoma Regional del Centro de Antioquia (Corantioquia) o su delegado, y el director del Área Metropolitana del Valle de Aburrá, entre otros.

Una ventaja es que el DAGRD opera en las mismas instalaciones que otras de las entidades mencionadas, especialmente las secretarías y despachos de la administración municipal, lo cual facilita la coordinación y articulación entre entidades al momento de la respuesta ante una emergencia.

\section{Comunicaciones autoridad-autoridad}

Las autoridades en Medellín no cuentan con un sistema tecnológico homogéneo para la comunicación, de manera que cada entidad mantiene su flujo de información, y la articulación entre los sistemas ha venido avanzando paulatinamente (Alcaldía Municipal de Medellín, 20I5). La
Alcaldía de Medellín cuenta con un sistema interno de correspondencia denominado Mercurio, y con las otras entidades, se interactúa in situ o por medios convencionales, como correo electrónico o teléfono fijo (Gómez \& Agudelo, 2019).

En una situación de emergencia, por la topografía de la ciudad, Medellín ha adquirido un sistema, CRONI, con frecuencias VHF y UHF abiertas a las cuales se han venido incorporando las entidades de la alcaldía, previendo en el futuro la incorporación de otras agencias, un tema que además de verse afectado por la disponibilidad presupuestal para la operación y el mantenimiento de las redes tiene que ver con la voluntad de las distintas autoridades para la circulación de información interconectada (Gómez \& Agudelo, 2019).

El Área Metropolitana ${ }^{4}$, a pesar de estar integrada por la ciudad de Medellín, cuenta con sistemas y redes diferentes que no facilitan la intercomunicación o la integración con el sistema CRONI, por costos, resultaría más beneficioso para la ciudad si los municipios del área metropolitana intervienen en este sistema y no a la inversa (Alcaldía de Medellín, 2015).

En definitiva, gran parte de las dificultades en la interoperabilidad de los sistemas y el flujo de información se ha solventado con la comunicación directa al contar con instalaciones contiguas dentro del mismo lugar.Así mismo, por el hecho de que la Ley 1523 faculte a cada entidad territorial para gestionar los procesos de la GRD, la Alcaldía de Medellín no requiere constantemente una comunicación directa con entidades del nivel departamental o nacional, $y$ en caso de ser necesario, se activa a través de los protocolos e instancias definidas.

\section{Comunicaciones individuo-autoridad}

Medellín, al igual que Bogotá, cuenta con un Número Único de Prevención Emergencias y Seguridad (123), el cual es financiado con recursos de la Secretaría de Seguridad y cuenta con personal de la alcaldía para apoyar la recepción de llamadas, lo que contribuye a aliviar la congestión en el sistema y a fortalecer un adecuado direccionamiento (Alcaldía de Medellín, 2015).

4 Integración de municipios del Valle de Aburrá: Caldas, La Estrella, Sabaneta, Itagüí, Envigado, Bello, Copacabana, Girardota, Barbosa y Medellín. 
Es importante resaltar que, aunque el 123 recibe llamadas únicamente de Medellín, la Policía, para temas de seguridad, actúa a nivel metropolitano, siendo la única entidad que tiene una cobertura metropolitana (Alcaldía de Medellín, 2015). Esta convergencia de responsabilidades y coberturas refleja parte de lo observado en el nivel nacional: el delegar el 123 a las responsabilidades de la Policía, con la complejidad de los requerimientos de seguridad en el país, no ha permitido un fortalecimiento de este como un sistema integral dentro del SNTE (Acosta, 2019; Rodríguez, 2019).

\section{Comunicaciones autoridad-individuo}

Medellín cuenta con una base sólida en conocimiento y reducción del riesgo, pues en 2013 el Instituto de Hidrología, Meteorología y Estudios Ambientales (Ideam), junto con el Departamento de Prevención de Desastres de la Gobernación de Antioquia (Dapard), lanzaron el primer Centro Regional de Alertas Tempranas del país, el Sistema de Alertas Tempranas del Valle de Aburrá (Siata). Esta herramienta permite tener un conocimiento de las condiciones hidrometeorológicas de la región, como alertas que se puedan generar por deslizamiento de tierra (Gobernación de Antioquia, 2013). Actualmente, el Siata permite la retroalimentación frente al reporte de nuevos eventos, y todas las entidades, tanto de la alcaldía como otras dependencias, pueden ingresar información que fortalece la detección temprana de amenazas y la comunicación oportuna a la comunidad (Alcaldía de Medellín, 2015).

Como complemento, se cuenta con el equipo social del DAGRD, “un grupo de profesionales del área social, que tienen un enlace directo con la comunidad, con los líderes comunitarios, con el voluntariado, con los comités barriales, los escolares, los institucionales, y a través de estas personas fluye la información" (Gómez \& Agudelo, 2019).

A través de la oficina de comunicaciones de la Alcaldía de Medellín y del DAGRD, se manejan las siguientes redes sociales: Facebook, Instagram, Twitter, página web, y allí se publican todas las campañas. Una gran dificultad en la actual administración municipal de Medellín ha sido la coordinación con la oficina de comunicaciones para la aprobación de los contenidos que se publican en la página (Gómez \& Agudelo, 2019), lo que ha incidido en la eliminación de algunos enlaces o páginas que permitían el acceso de la ciudadanía en general a información y, sobre todo, a la documentación del DAGRD.

El caso de Medellín, al igual que Bogotá, presenta un desarrollo tecnológico e institucional que ha permitido sortear las necesidades de telecomunicaciones en emergencias dentro del ámbito local (Gómez \& Agudelo, 2019). Para lograr su articulación con las otras entidades territoriales en un SNTE, requieren plataformas interoperables en el resto del país que permitan la integración con sus propias redes, más allá de la adquisición de nuevas infraestructuras u operadores (Acosta, 2019). La categoría de comunicaciones individuo-individuo no presenta acciones por parte de las autoridades en Medellín. La intervención directa, en este caso, es competencia de las autoridades nacionales, especialmente el MinTIC.

\section{Conclusiones y recomendaciones}

Es evidente el avance normativo en el ámbito nacional para reglamentar el SNTE, pero aún no está implementado en Bogotá y Medellín. Estas ciudades cuentan con recursos financieros, técnicos y físicos, y han desarrollado sus propios sistemas incluso antes de la implementación del SNTE, aunque estos no tengan como objetivo la ampliación de servicios hacia otras entidades territoriales, en busca de una mayor articulación.

Además, con base en las entrevistas a los expertos, ambas ciudades reivindican la competencia de la administración municipal para manejar de manera autónoma, por descentralización, sus procesos de GRD y las comunicaciones inmersas; si bien presentan dificultades, como cualquier otro municipio o departamento, consideran que estas pueden solucionarse en el nivel local y no requieren una reglamentación adicional (Gómez \& Agudelo, 2019;Vargas, 2019). En cuanto a sus sistemas de alerta temprana, los desarrollos con los que cuentan las ciudades de Bogotá y Medellín se consideran una referencia para el resto del país, aunque se reconoce que los recursos de estas dos entidades territoriales son muy superiores al común denominador de los municipios en Colombia.

Observando, entonces, el avance de las ciudades objeto de estudio, es posible concluir que, desde el nivel nacional se requiere una regulación que permita la conectividad con otras redes existentes en el país, que facilite la interoperabilidad y la disposición presupuestal para llevarla a cabo, sin que se incurra en una saturación para las obligaciones de las entidades locales, o una interferencia con las competencias establecidas en la Ley 1523.

En el ámbito local, las dificultades y problemáticas observadas tienen eco a nivel nacional, pues se resaltó la falta de articulación entre entidades para coordinar la adquisición de equipos y sistemas interoperables, la saturación 
de la línea de emergencias y la necesidad de brindar una institucionalidad que permita el apoyo a la Policía Nacional para el adecuado direccionamiento de las llamadas.

Al respecto, el MinTIC cuenta con recursos, capacidades, infraestructura y capital humano que permitiría el desarrollo del SNTE sin interferir con las competencias municipales, y actualmente cuenta con el apoyo e interés de las otras entidades nacionales, lo cual garantizaría su implementación oportuna. El concurso de entidades, como el Departamento Nacional de Planeación, para buscar la financiación del SNTE, es un avance que, sin duda, potencia los avances de las ciudades de estudio y permite la réplica de medidas exitosas para el resto de municipios del país.

Hay dos grandes desafíos políticos, el primero es lograr que la información del SNTE sirva para definir esquemas de gobernabilidad de la gestión del riesgo para las ciudades (Botero, Arrizabalaga, Milanés, \&Vivas, 20I7), y el otro es la inclusión de los actores sociales y de sus redes para reducir el escenario de vulnerabilidad social vinculado con los desastres. (Vivas Cortés, Lampis, Romero, Delgado, \& Franco Castro, 2017).

\section{Reconocimientos}

Artículo de presentación de resultados de la investigación sobre la implementación del Sistema Nacional de Comunicaciones en Emergencias: casos de Bogotá y Medellín.

\section{Referencias}

Acosta, J. (10 de febrero de 2019). Entrevista a exfuncionario de MinTIC. (G.A. Rico, entrevistador).

Alcaldía de Medellín. (20I5). Plan municipal de gestión del riesgo de desastres de Medellín.

Alcaldía Mayor de Bogotá. (20।6). Plan de desarrollo: Bogotá mejor para todos. Bogotá, DC.

Alcaldía Municipal de Medellín. (2015). Plan municipal de gestión del riesgo de desastres de Medellín.

Alcaldía Municipal de Medellín. (2016). Plan de Desarrollo Medellín cuenta con vos, 2016-2019. Medellín.

Agencia Nacional del Espectro (ANE). (2017). Propuesta de banda angosta para atención de emergencias y desastres en Colombia. Recuperado de https://www.ane.gov.co/ images/ArchivosDescargables/consultapublica/PropuestaBandaAngostaEmergenciasyDesastres/Proyecto_banda_angosta.pdf
Agencia Nacional del Espectro (ANE). (noviembre de 2018). Respuesta a comentarios de MinTIC al proyecto Propuesta banda angosta para atención de emergencias y desastres en Colombia. Recuperado de https://www. ane.gov.co/images/ArchivosDescargables/consultapublica/DocumentoRespuestaBandaAngosta/Respuestas_comentarios_MINTIC_Proyecto_Emergencias_R2.pdf

Ávila Toscano, J., Vivas Cortés, Ó., Herrera Flórez, A., \& Jiménez, M. (2016). Gestión del riesgo de desastres en el Caribe Colombiano desde la óptima de organismos de socorro y administraciones locales: el caso del sur del Atlántico. Revista Luna Azul (42), 68-88. http://dx.doi. org/I0.17I5I/luaz.2016.42.7

Botero Saltarén, C., Arrizabalaga Fal, M., Milanés Batista, C., \& Vivas Cortés, O. (2017). Indicadores de gobernabilidad para la gestión del riesgo costero en Colombia. Revista Luna Azul (45), 227-25I. http://dx.doi.org//0.17/5I/ luaz.2017

Comisión de Regulación de Comunicaciones (CRC). (2016). Resolución 4972. Bogotá.

Comisión de Regulación de Comunicaciones (CRC). (2016). Regulación para promover el desarrollo e implementación del Sistema Nacional de Telecomunicaciones de Emergencias en Colombia. Bogotá.

Congreso de la República de Colombia. (2009). Ley I34I. Recuperado de http://www.mintic.gov.co/portal/604/articles-3707_documento.pdf

Congreso de la República de Colombia. (2012). Ley I523. Bogotá.

Decreto 1240. (2015).Alcaldía Municipal de Medellín. Medellín.

Decreto 172. (2014). Alcalde Mayor de Bogotá. Bogotá.

Departamento Administrativo Nacional de Estadística (DANE). (2018). Resultados preliminares Censo Nacional de Población y vivienda. Bogotá.

Díaz, J., Chuquisengo, O., \& Farradas, P. (2005). Manual de gestión de riesgo en los gobiernos locales. Lima: Soluciones prácticas.

Departamento Nacional de Planeación (DNP). (2014). Misión Sistema de Ciudades. Bogotá.

Departamento Nacional de Planeación (DNP). (2018). Desarrollo territorial. Tipologías municipales. Recuperado de www.dnp.gov.co

El Tiempo. (20 de octubre de 2018). Bromas a la línea 123 serán sancionadas. Recuperado de https://www.eltiempo.com/bogota/personas-que-hagan-bromas-en-linea|23-serian-multados-I I0430 
Fondo de Tecnologías de la Información y las Comunicaciones- Fontic. Consorcio Itelca-STI. (2013). Diseño de la red nacional de telecomunicaciones de emergencias y establecimiento de un marco normativo para el fortalecimiento del sistema nacional de telecomunicaciones de emergencias en Colombia. Bogotá: MinTIC.

Fondo de Tecnologías de la Información y las Comunicaciones- Fontic. Iteco. (2013). Identificar y analizar las buenas prácticas en relación con las redes nacionales de telecomunicaciones de emergencias y desastres. Bogotá.

Gobernación de Antioquia. (2013). CRPA. Recuperado de http://antioquia.gov.co/index.php/alertas-tempranas

Gómez, A., \& Agudelo, S. (27 de febrero de 2019). Entrevista a funcionarios del Departamento Administrativo de Gestión del Riesgo de Desastres (DAGRD). (G. A. Rico, entrevistador).

Instituto Distrital de Gestión de Riesgos y Cambio Climático (Idiger). (2015). Plan Distrital de Gestión de Riesgos y Cambio Climático para Bogotá, 2015-2050. Documento técnico de soporte. Bogotá:Alcaldía Mayor de Bogotá.

Instituto Distrital de Gestión de Riesgos y Cambio Climático (Idiger). (2016).Acuerdo 007. Bogotá.

Instituto Distrital de Gestión de Riesgos y Cambio Climático (Idiger). (2017a). Elaboración de lineamientos para el manejo de emergencias. Bogotá.

Instituto Distrital de Gestión de Riesgos y Cambio Climático (Idiger). (20I7b). Coordinación de Servicios de respuesta, emergencias y desastres. Protocolo CAE-PD-05 versión 6. Bogotá.

Medina, F. (20I4). La gestión del riesgo y las políticas de cambio climático en la agricultura ecológica. Almería: Universidad de Almería.

Ministerio de las Tecnologías de la Información y las Comunicaciones (MinTIC). (20I4). Decreto 2573. Recuperado de Por el cual se establecen los lineamientos generales de la Estrategia de Gobierno en línea: http://www.mintic.gov. co/portal/604/w3-article-I4455.html

Ministerio de las Tecnologías de la Información y las Comunicaciones (MinTIC). (20I5). Decreto 2434 de 2015. Recuperado de http://www.alcaldiabogota.gov.co/sisjur/ normas $/$ Normal.jsp?i=642 18

Ministerio de las Tecnologías de la Información y las Comunicaciones (MinTIC). (2016). Política Pública del Sistema Nacional de Telecomunicaciones en Emergencias.Versión I.0. Bogotá
Ministerio de las Tecnologías de la Información y las Comunicaciones (MinTIC). (2018). Información sobre los planes de mejoramiento. Recuperado de https://www.mintic.gov. co/.../articles-4327_plan_mejoramiento_ministerio_tic

Ortiz, L., \& Valencia, F. (2017). Gestión de riesgos en eTOM. Un análisis comparativo con los estándares de riesgo corporativo. Revista Logos Ciencia \& Tecnología, 9(I), 85-99. http://dx.doi.org//0.22335/rlct.v9il.334

Rodríguez,J. (20 de febrero de 2019). Entrevista a funcionario MinTic. (G.A. Rico, entrevistador)

Unidad Nacional para la Gestión del Riesgo de Desastres (UNGRD). (2015a). Sistema Nacional de Gestión del Riesgo de Desastres. Anexo I. Diagnóstico de la gestión del riesgo de desastres. Bogotá.

Unidad Nacional para la Gestión del Riesgo de Desastres (UNGRD). (2015b). Plan nacional de gestión del riesgo de desastres 2015-2025. Bogotá.

Unidad Nacional para la Gestión del Riesgo de Desastres (UNGRD). (20I5c). Subdirección de Manejo de Desastres. Recuperado de Desastres: http://portal.gestiondelriesgo.gov.co/Paginas/Manejo_de_Desastres.aspx

Unidad Nacional para la Gestión del Riesgo de Desastres (UNGRD). (2019). Fondo Nacional de Gestión del Riesgo de Desastres. Recuperado de http://portal.gestiondelriesgo.gov.co/Paginas/Fondo-Nacional-de-Calamidades.aspx

Unión Internacional de Telecomunicaciones (UIT). (2003). Recomendación UIT-T E. 106: Plan internacional de preferencias en situaciones de emergencia para actuaciones frente a desastres. Recuperado de https://www.itu.int/ rec/T-REC-E.106-200310-I/es

Vargas, R. (19 de enero de 2019). Entrevista al director general del Idiger Bogotá. (G.A. Rico, entrevistador)

Vivas Cortés, Ó., Lampis, A., Romero Huertas, C., Delgado, P., \& Franco Castro, C. (2017). Lineamientos para el análisis de la vulnerabilidad social en los estudios de la gestión del riesgo municipal de desastres. Bogotá: UNGRD. Recuperado de https://repositorio.gestiondelriesgo.gov.col bitstream/handle/20.500.I 1762/24756/LAVS_Web-.pdf? sequence $=\mid$ \&isAllowed $=y$ 